\title{
Ultra-Broadband Dielectric THz Spectroscopy with Air-Biased-Coherent-Detection
}

\author{
D'Angelo, Francesco; Bonn, Mischa; Turchinovich, Dmitry
}

Published in:

Proceedings of the 38th International Conference on Infrared, Millimeter and Terahertz Waves IRMMW-THz 2013

Link to article, DOI:

10.1109/IRMMW-THz.2013.6665505

Publication date:

2013

Link back to DTU Orbit

Citation (APA):

D'Angelo, F., Bonn, M., \& Turchinovich, D. (2013). Ultra-Broadband Dielectric THz Spectroscopy with AirBiased-Coherent-Detection. In Proceedings of the 38th International Conference on Infrared, Millimeter and Terahertz Waves IRMMW-THz 2013 IEEE. https://doi.org/10.1109/IRMMW-THz.2013.6665505

\section{General rights}

Copyright and moral rights for the publications made accessible in the public portal are retained by the authors and/or other copyright owners and it is a condition of accessing publications that users recognise and abide by the legal requirements associated with these rights.

- Users may download and print one copy of any publication from the public portal for the purpose of private study or research.

- You may not further distribute the material or use it for any profit-making activity or commercial gain

- You may freely distribute the URL identifying the publication in the public portal 


\title{
Ultra-Broadband Dielectric THz Spectroscopy with Air-Biased- Coherent-Detection
}

\author{
Francesco D'Angelo ${ }^{1}$, Mischa Bonn ${ }^{1}$, and Dmitry Turchinovich ${ }^{1,2}$ \\ ${ }^{1}$ Max Planck Institute for Polymer Research, 55128 Mainz, Germany \\ ${ }^{2}$ DTU Fotonik, Technical University of Denmark, 2800 Lyngby, Denmark
}

\begin{abstract}
We present results on ultra-broadband THz-TDS on silicon using air biased coherent detection (ABCD) technique. We find that the positioning of the sample in the spectrometer, leading to the spatial shift of THz focus, is crucial for accurate spectroscopy results.
\end{abstract}

\section{INTRODUCTION AND BACKGROUND}

$\mathrm{T}$ he current most established heterodyne ultra-broadband $\mathrm{THz}$ detection in gaseous media is called ABCD (Air Biased Coherent Detection) [1]. In spite of an easy access to the high ( $>15 \mathrm{THz}$ ) frequencies using $\mathrm{THz}$ air photonics generation and detection, only a few reports on ultrabroadband $\mathrm{THz}$ time-domain spectroscopy (THz TDS) using $\mathrm{ABCD}$ have been published [2].

Here, we report transmission-mode THz-TDS on intrinsic silicon (1 mm thick, double side polished wafer) using ABCD. By analyzing the transmitted reference and sample $\mathrm{THz}$ pulses, we calculated the absorption and refractive index spectra. We found that the results of the spectroscopy are strongly affected by the position of the sample in the spectrometer.

\section{RESULTS}

Our setup is shown in Fig. 1. We used a Ti:Sapphire amplified laser system which produces 35 fs pulses at $800 \mathrm{~nm}$ central wavelength, with $1 \mathrm{kHz}$ repetition rate. The beam was split into pump $(600 \mu \mathrm{J}$ pulse energy) for $\mathrm{THz}$ generation, and probe $(50 \mu \mathrm{J}$ pulse energy) pulses for $\mathrm{ABCD}$ detection. The pump beam was frequency-doubled using a $\beta$-BBO crystal $(0.1 \mathrm{~mm}$ thick). Both the second harmonic $(\mathrm{SH})$ and the fundamental beams were then focused together in air using a $200 \mathrm{~mm}$ focal length lens.

The high peak intensity achieved in the focal region by the 2-color laser field induces an ultrafast ionization of air, resulting in a plasma filament. The laser field in the focus has a special temporal profile due to the presence of both $400 \mathrm{~nm}$ SHG signal and $800 \mathrm{~nm}$ fundamental of the laser. If the relative phase between the laser fundamental and second harmonic is adjusted properly, the free electrons in the ionized plasma are driven preferentially in one direction, thus producing a transient current which radiates at the $\mathrm{THz}$ frequencies (essentially difference frequency generation between the $400 \mathrm{~nm}$ field and the overtone of the $800 \mathrm{~nm}$ field). A detailed description of ultra-broadband $\mathrm{THz}$ emission by dual-color femtosecond laser filamentation in air can be found in reference [3].

The filament-emitted $\mathrm{THz}$ radiation was collimated and then focused at the sample position B by two $90^{\circ}$ off axis parabolic silver mirrors (OPM). The transmitted $\mathrm{THz}$ radiation is again collimated, and re-focused by a second couple of OPM to a commercial ABCD detector (Zomega THz Corp). The ABCD detection is based on the principle of $\mathrm{THz}$ electric field induced SHG in air. Air possesses a non-zero third-order susceptibility $\chi^{(3)}$ air, however the natural second-order susceptibility of air is zero because of its symmetry. In the presence of external electric bias, such as e.g. by the electric field of the $\mathrm{THz}$ pulse $\mathrm{E}_{\mathrm{THz}}$, an effective second-order susceptibility is produced $\chi^{(2)}{ }_{\text {air }}=\mathrm{E}_{\mathrm{THz}} \chi^{(3)}$ air, which in turn allows for frequency-doubling the laser pulse co-propagating with the THz field. The intensity of the measured SHG signal is proportional to the $\mathrm{THz}$ field strength $\mathrm{E}_{\mathrm{THz}}$. The phase of the $\mathrm{THz}$ signal can be determined by applying a static (high) voltage to the air, which is provided by the pair of electrodes in the $\mathrm{ABCD}$ detector. The externally-modulated static voltage enters into the mixing of the laser and $\mathrm{THz}$ fields as a local oscillator signal.

The efficiency of ABCD detection is thus dependent on optimal co-propagation of the $\mathrm{THz}$ signal and a probe laser pulse in the space between bias electrodes of the ABCD detector. In our setup, both the $\mathrm{THz}$ and the probe beam were focused together in the spacing between the two high-voltage electrodes $( \pm 1.5 \mathrm{kV}$ at $500 \mathrm{~Hz})$ as shown in the inset of Fig. 1 . The time delay between the probe and the $\mathrm{THz}$ pulse was controlled by a standard optical delay line. The experiment was carried out in purged air in order to avoid strong $\mathrm{THz}$ absorption due to atmospheric water vapor.

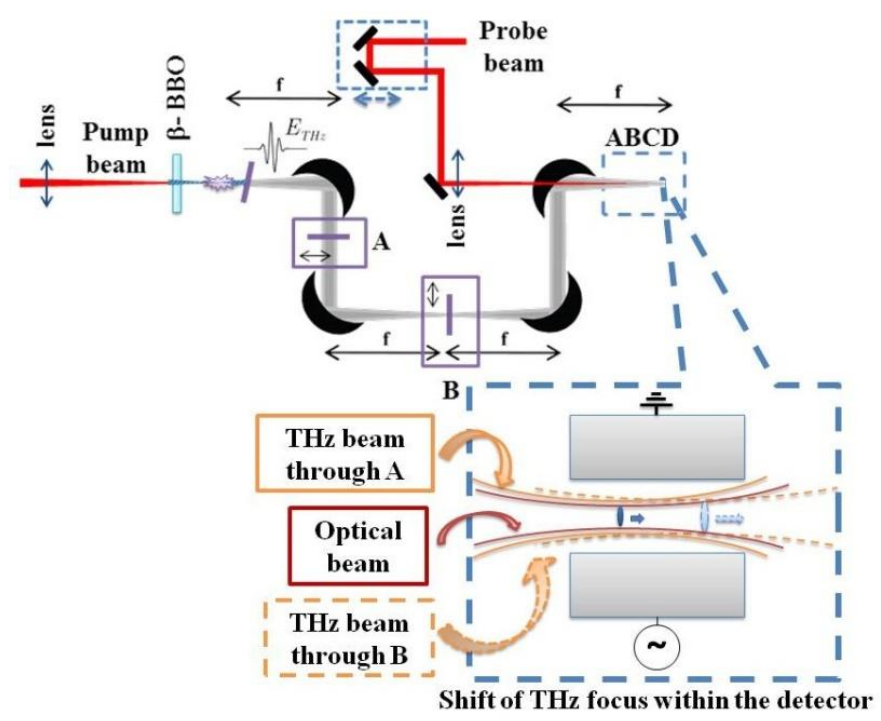

Fig. 1: Ultra-broadband THz-TDS set-up. The THz focus is shifted forward in the detector when the sample is placed in B instead of A. 
In our THz TDS measurements on silicon we acquired the transmitted $\mathrm{THz}$ pulse through our silicon sample in two configurations: when the sample was placed in the collimated $\mathrm{THz}$ beam (position A), and when it was placed in the focused THz beam (position B).

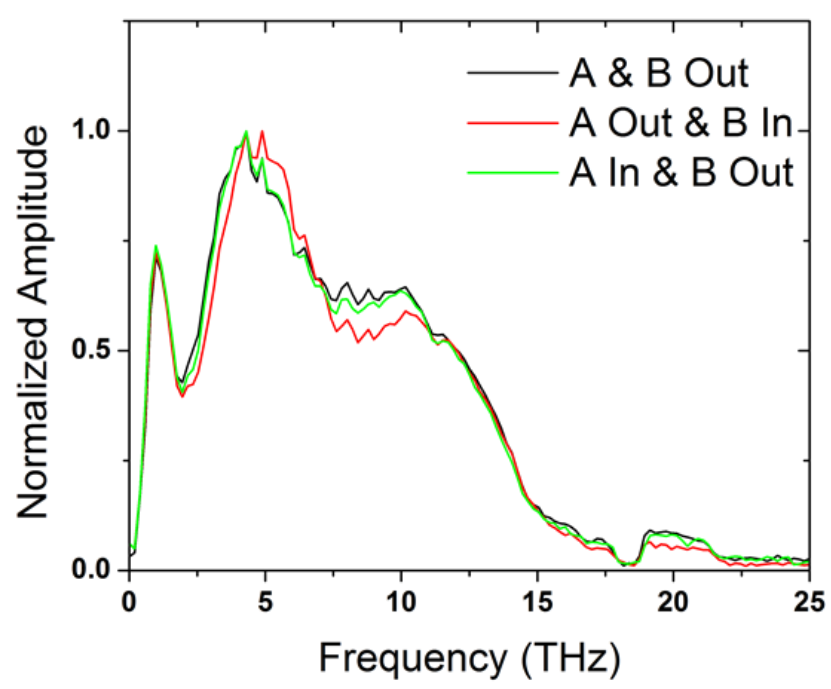

Fig. 2: Normalized $\mathrm{THz}$ spectra at sample positions in the collimated beam (green) and in the focus (red). Black line is the normalized reference spectra.

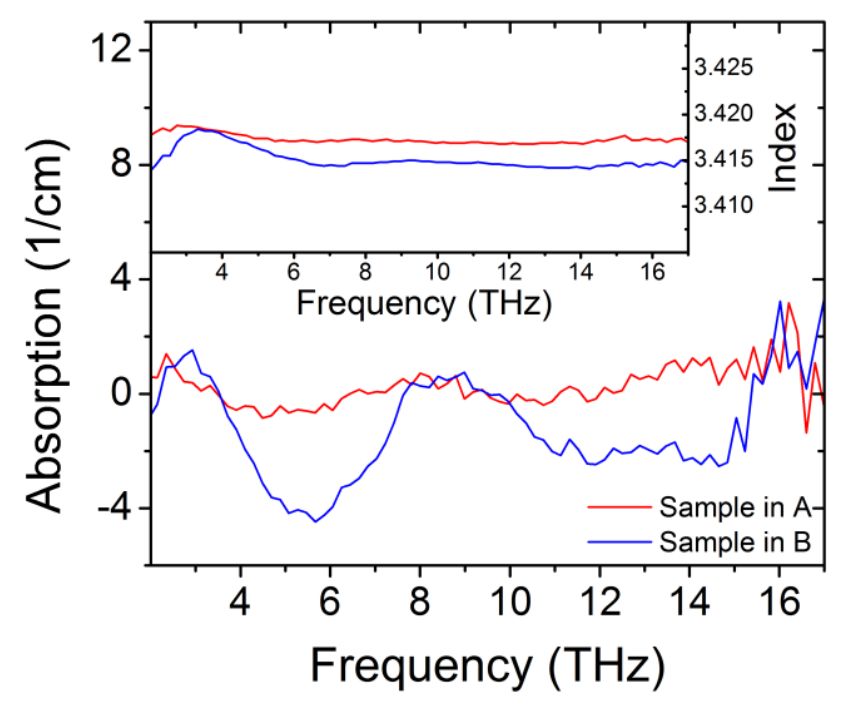

Fig. 3: Frequency dependent absorption coefficient and index of refraction of silicon in the case the sample is positioned in the collimated section of the $\mathrm{THz}$ beam (A) and in the focal point (B) of the spectrometer.

By analyzing the transmitted reference and sample $\mathrm{THz}$ pulses, we calculated the absorption and refractive index spectra in these two experimental arrangements. It is evident from the amplitude spectra of TDS reference and sample signals which are shown in Fig. 2, that the measured traces depend on the precise position of the sample. Although the $\mathrm{THz}$ field strength is different in the two positions, we can rule out non-linear effects that may be present for higher field strengths, as even in the focus (position B), the field strengths are insufficient for non-linear $\mathrm{THz}$ optical effects. Nonetheless, we found a strong effect of the sample positioning on the measured absorption spectrum; the measured refractive index spectrum which is only dependent on the phase spectra of reference and sample signals was less affected. In Fig. 3 we show the resulting inferred frequencydependent absorption and index of silicon in the case when the sample was placed in the focal point of the spectrometer (B), and in the case when it was positioned in the collimated beam (A). It is apparent that the spectroscopy results for the case of the sample in the focal point become physically meaningless, featuring negative absorption (i.e. gain) in un-doped silicon at $\mathrm{THz}$ frequencies. When the sample is positioned in the collimated beam, the ultra-broadband spectroscopy results are indeed consistent with the low-THz frequency data [4] featuring near-zero absorption and spectrally-flat refractive index of $n \approx 3.418$.

The THz-TDS analysis is based on the assumption of the plane waves. This assumption is valid if the sample is positioned either in the collimated beam, or precisely in the focal point of the spectrometer (assuming that the sample is infinitely thin). However, positioning a finite-thickness sample in the focal point of the spectrometer will spatially translate the image of the sample position in the detector, as shown in inset of Fig. 1. We found that the observed amplitude spectrum modulation, leading to apparent "gain", is caused by is this spatial shift of the focus of the $\mathrm{THz}$ beam in the detector, as was also discussed in [5,6]. ABCD is based on copropagation of perfectly phase matched and spatially overlapped optical probe and $\mathrm{THz}$ beams. Since the focus of the $\mathrm{THz}$ beam in the detector is shifted, the overlap between the two beams in the detector can drastically change, leading to fringes in $\mathrm{THz}$ spectrum (see red line in Fig. 2), only appearing in the presence of the sample in the focal point of the spectrometer.

In summary, we demonstrate that the positioning of the sample in the transmission-mode THz-TDS spectrometer based on ABCD detection is crucial for meaningful spectroscopy. The correct spectroscopy results can only be achieved if the imaging of the $\mathrm{THz}$ beam in the detector is not disturbed by the presence of the sample, e.g. by positioning the sample in the collimated part of the $\mathrm{THz}$ beam.

\section{REFERENCES}

[1] N. Karpowicz et al., Appl. Phys. Lett. 92, 011131 (2008).

[2] N. Vieweg et al., Opt. Express 20, 28249 (2012).

[3] K.Y. Kim et al., Opt. Express 15, 4577 (2007).

[4] D. Grischkowsky et al., J. Opt. Soc. Am. B 7, 2006 (1990).

[5] P. Kužel et al., Opt. Express 18, 15338 (2010).

[6] K. Iwaszczuk and P. U. Jepsen, paper Th2-40, in Proc. of International workshop of Optical Terahertz Science and Technology, Kyoto (2013). 